\title{
A Position Sensitive Clustering Algorithm for VANET
}

\author{
http://dx.doi.org/10.3991/ijoe.v10i1.3145 \\ H.Q. Liu, L.C. Yang*, Y. Zhang and L. Wu \\ School of Control Science and Engineering, Shandong University, Jinan, Shandong, China
}

\begin{abstract}
In order to overcome the defects such as too many cluster heads and large overhead in traditional single hop VANET clustering algorithms when there are high density vehicles, this article proposes a multi-hop cluster algorithm named the Position Sensitive Clustering Algorithm (PSCA). Based on the establishment of cluster structure, initial cluster head is selected following the 'Randomly Competition, First Declare Win' principle. Cluster members transmit their expected status messages through the maintained multiple hop routings. After a series of information fusion and calculation work, the cluster head broadcasts the management messages based on dynamic timestamps. This article also gives the cluster maintaining strategy when the cluster head disappears or new nodes join. Tests performed in the simulation by NS-2 show that the proposed algorithm has less cluster heads and lower control overhead compared to the single hop-based approach (the Lowest-ID algorithm).
\end{abstract}

Index Terms-clustering, VANET, geography position, dynamic timestamp

\section{INTRODUCTION}

Vehicular Ad-hoc Network (VANET) is a special case of Mobile Ad-hoc Network (MANET) which contains vehicle to vehicle communications and vehicle to roadside-unit communications in traffic environment. VANETs indicate the development trend of Intelligent Transportation System (ITS). By the study and implementation of VANETs, several practical traffic applications are enabled [1-3], for example, vehicle safety information release, road traffic coordination control, high real-time traffic information collection and traffic route guidance. However, compared to MANETs, unique characteristics of VANETs impose many challenges and constraints to implement these applications. There are always large numbers of vehicles in the communication environment, which are distributed on different roads. And the movements are restricted by the topological of road network. The variation of speed and density both in the time and spatial scales may result in frequent changes of network topology, low real-time and poor scalability. Although the VANETs have such challenges and constraints, the VANETs have some advantages. For example, nodes in VANETs have more processing power and storage space, and they can obtain some extra information such as position, speed and acceleration through the on-board equipments [4].

Clustering in VANET is nothing but organizing vehicles themselves into groups according to some specific common characteristics. Cluster head is selected among vehicles and is responsible for coordinating the members of the cluster [5]. Clustering offers a kind of hierarchical organization to provide scalability and basic performance guarantee by partitioning the network into disjoint groups of nodes [6]. Clustering is of great significance in VANET. For example, cluster-based routing can optimize the bandwidth utilization, reduce the overhead of route discovery and maintenance and improve real-timing. What's more, some Cooperative Vehicle Infrastructure System (CVIS) applications will be facilitated. Cluster head is responsible for the collection and release of traffic status information, therefore computational cost of information center will be reduced.

The aim of clustering in VANET is to establish a certain virtual structure before application data transmitting. Cluster heads and gateways form a backbone to facilitate intra-cluster and inter-cluster transmission, hence increasing spatial channel reuse and maximizing network capacity[7-8]. A good clustering algorithm for VANET should not only be stable when the topology changes, but also has lower cluster maintenance overhead and less cluster heads in the network. In order to overcome the defects such as too many cluster heads and large overhead in traditional single hop VANET clustering algorithms when vehicle density is high, this article proposes a Position Sensitive Clustering Algorithm (PSCA) based on configured road section position information and dynamic timestamps. In PSCA, the cluster head releases management message at a certain timestamp by direction-based broadcasting. Cluster members deliver their expected status information before the next timestamp by the stored routing to the cluster head. The cluster heads' initial selection follows the principle of 'Randomly Competition, First Declare Win'. The PSCA also gives the maintenance strategy for the cases of cluster head disappearing and new nodes' joining. In order to justify the proposed algorithm, a simulation is deployed using NS-2. Compared with the Lowest ID algorithm, it has less cluster heads and lower control overhead.

The rest of the paper is structured as follows. Section 2 presents an overview of the existing clustering algorithms in VANET. Section 3 presents the process of the cluster establishment and initial cluster head selection. In Section 4, a cluster maintenance strategy based on dynamic timestamps is presented. Section 5 introduces some other principles and analyzes the performance of the proposed algorithm. Taking the number of cluster heads and data packages as evaluation index, a simulation is given compared to Lowest-ID algorithm in Section 6. At last, we make a conclusion and discuss the future work.

\section{RELATED WORK}

The purpose of clustering algorithm in VANET is to create a stable, highly extensible and low cost cluster structure so that it can better serve the related applications. 
Since the VANET is a case of MANETs, traditional clustering methods for MANETs are still suitable, such as Lowest-ID algorithm [9]. The Lowest-ID is a classic clustering algorithm, in which nodes broadcast HelloMessages containing their ID data periodically, so that a table of neighbor nodes' ID information could be formed. The node which has the lowest ID will be selected as the cluster head and the rest are cluster members.

In [10], the author proposes a weighted utility function (WUF) for VANET clustering based on weighted clustering algorithm [11]. Vehicle ID, node degree and other traffic index such as average speed, distance between nodes are selected as parameters of the function and each parameter is assigned as a certain weighting factor. Vehicle mobility is taken into consideration, so the algorithm shows good performance on stability and load-balancing. However, when relative position frequently changes, for example, in the relative movement environment, it will reduce the stability and add more overhead.

In [12], a robust mobility adaptive clustering scheme for VANET is given. It employs a novel node precedence algorithm to adaptively identify the nearby single hop neighbors and selects optimal cluster heads based on relative node mobility metrics of speed, locations and directions. It also introduces the zone of interest concept for the optimized approach to the network structure. It is very effective in the highly dynamic VANETs environment and robust on link failures. But it also causes high overhead.

Reference [13] proposes a cluster formation scheme based on direction of vehicle at the approaching intersection and the node which is the first vehicle moving in a particular direction is selected as the cluster head. It has a defect that more overhead will be created if the cluster head changes frequently due to nodes moving at variable speed.

Since vehicles can get their geography positions by using GPS, some position-based clustering algorithms have been proposed. In [14], a position-based clustering technique is presented. The cluster structure is determined by the geography positions of nodes and the priorities associated with the vehicle information. A node becomes a cluster head should satisfies two conditions: 1) The node has the highest priority in its single hop neighborhood, and 2)it has the highest priority in the single hop neighborhood of one of its single hop neighbors. Because the size of a cluster is established beforehand, it will cause extra overhead for cluster head's reselection when vehicle density changes. In the algorithm proposed in [15], each road is divided into segments and in each segment some anchor points are defined. Vehicles near the anchor points are selected as cluster heads. But this algorithm fails to address the cluster maintenance and stability issues.

Clustering algorithms for VANET presented above are almost single hop-based, in other words, the distance between the cluster head and a cluster member is only one hop. These algorithms can be achieved easily through each vehicle broadcasting hello-messages to its neighbors. However, limited by the communication range, there are only a few nodes in each cluster. This causes some problems. On one hand, computing and network resources will not be fully utilized. On the other hand, there will be more clusters when the network size is large. And more overhead will be created if the number of vehicles or the traffic density increases. In the MANETs field, scholars have proposed some multi-hop clustering algorithms [16,17], while in VANET, related study is scarce. Multi-hop cluster algorithm can effectively decrease the number of clusters so that utilization of wireless channels can be raised and transmission delay will be reduced.

\section{Cluster Establishment And Initial Cluster HEAD SELECTION}

Clustering can be normally classified into two stages, cluster formation and cluster maintenance [18]. In this section, we will introduce the cluster formation stage of PSCA.

\section{A. Assumptions and a Definition}

Firstly, we give some necessary and rational hypotheses and a definition.

1. Each vehicle is equipped with GPS, wireless communication facility and other hardware equipments, which can help to obtain its location, speed and accelerated speed. Vehicles exchange data with neighbors using shortrange wireless communication. Furthermore, vehicle can obtain accurate time information through the time synchronism service provided by GPS.

2. Clustering management messages are broadcasted and forwarded with the highest priority. When the hop count is few, influences on the algorithm caused by forwarding delay will be neglected.

3. Links between vehicles are bi-directional, in other words, if node $a$ is able to send message to node $b$, node $b$ can also make a connection with a.

Definition Timestamp: A timestamp in the clustering algorithm is the broadcasting time point for the cluster head sending its management message to all members.

\section{B. Cluster Establishment}

All vehicles record the database of geography position information of the one-way traffic roads in the network, which have been divided into different sections. Each section establishes a certain VANET cluster. According to the direction of the road, there will be two different clusters if it is two-way road. Vehicles entering into the geography area of a certain cluster become members of the cluster automatically. There are three kinds of roles of the vehicle nodes, which are cluster head $(\mathrm{CH})$, cluster member (CM) and cluster gateway (CG).

$\mathrm{CH}$ : The cluster head is responsible for the cluster maintenance. It records status information of all its members. In a cluster, there is at least one cluster head.

CM: Nodes become cluster members only if they enter into the geography area of a cluster. All members maintain the routing information to the cluster head.

CG: Vehicles in the overlapping area of different clusters are gateway nodes. They are special parts of cluster members. Adjacent clusters exchange information through the gateway nodes. ure.1.

The cluster establishment process is described in Fig- 
SPECIAL FOCUS PAPER

A Position Sensitive Clustering Algorithm for VANET

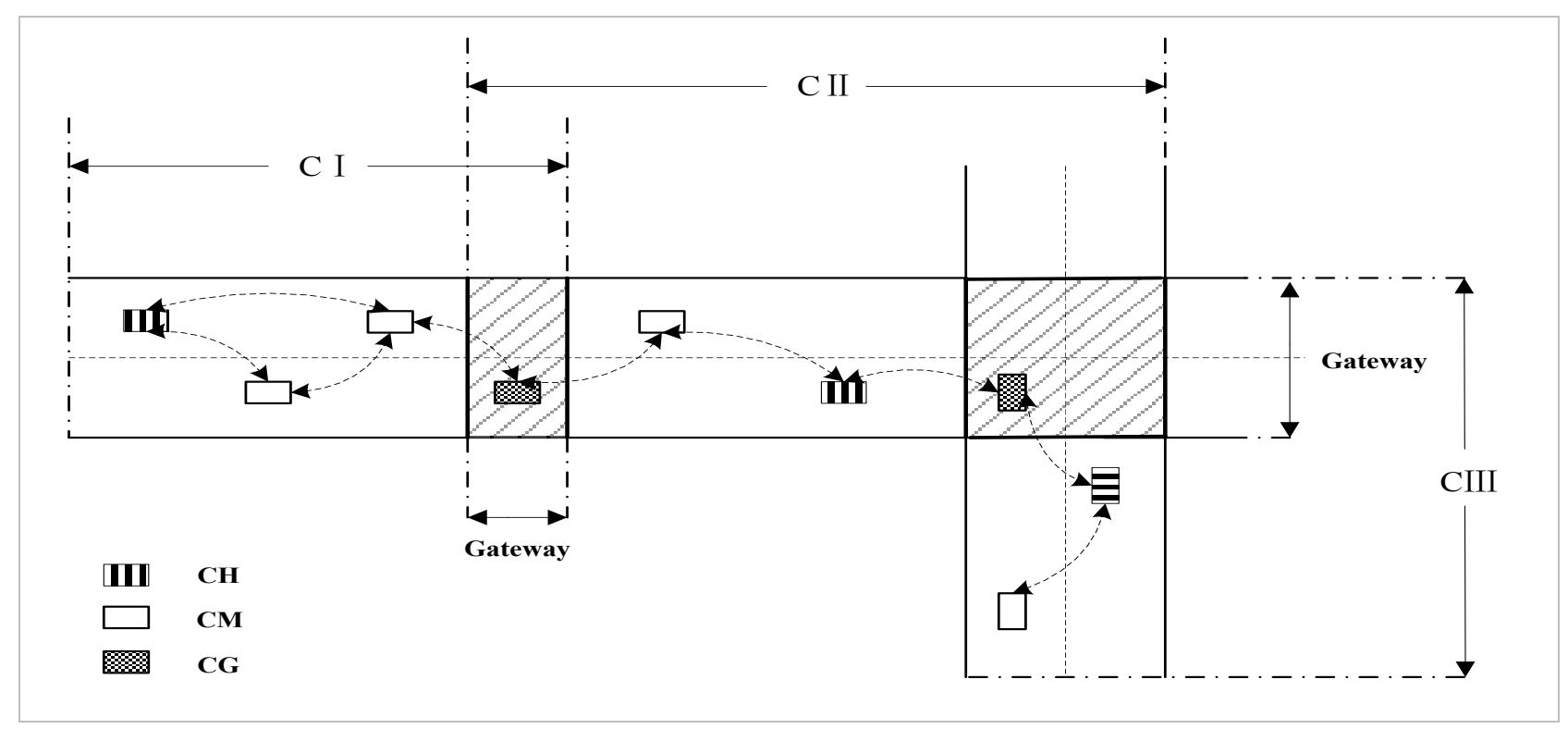

Figure 1. Establishment of the cluster structure based on road position

\section{Initial Cluster Head Selection}

In the stage of initial cluster head selection, all nodes haven't got the cluster's status information. The selection of initial cluster head follows the 'Randomly Competition, First Declare Win' principle. All nodes generate a random number $\mu(i)$, which is in the range of $[0,1]$. Then each node delays a time interval $\mu(i) \bullet \sigma_{\max }$ according its ran-

dom number, where $\sigma_{\max }$ denotes the maximum delay time. During the time interval, if the node hasn't received any cluster head declaration message, it declares itself as the cluster head through multi-hop broadcasting to all members. The format of Declaration-Message is designed as follows:

$$
<I D_{-} C H, L_{-} C H, I D_{-} F W, L_{-} F W, T_{0}, K>
$$

Where $I D_{-} C H$ is the cluster head's ID and $L_{-} C H$ is its location. $I D_{-} F W$ and $L_{-} F W$ are the ID and location information of nodes who forward the message. $T_{0}$ is the initial timestamp. $K$ is the hop count.

\section{Cluster Maintenance Scheme Based On DynamiC TIMESTAMPS}

Once the cluster structure is established, maintenance should be indispensable for the reason that vehicles move at variable speed, causing frequent changes of network topology. In this part, we present a cluster maintenance scheme based on dynamic timestamps.

\section{A. Direction Based Broadcasting Scheme and Routing Establishment}

Cluster head broadcasts management messages. Cluster members receive them and forward timely. In traditional flooding algorithm, all nodes forward the same message and this will result in large amount of duplicate messages, causing the broadcast storm problem. By making the most of position information obtained by GPS, this article proposes a direction based broadcasting algorithm for cluster management messages' release.
The cluster management message contains position information of the cluster head and forwarding node. When a node receives the message, it calculates the distance between itself and the cluster head $\operatorname{dis}(\operatorname{Node}(i), \mathrm{CH})$, as well as the distance between the latest forwarding node and the cluster head $\operatorname{dis}\left(\right.$ Forward $\left._{\text {latest }}, \mathrm{CH}\right)$. If $\operatorname{dis}(\operatorname{Node}(i), \mathrm{CH})>\operatorname{dis}\left(\right.$ Forward $\left._{\text {latest }}, \mathrm{CH}\right)$, the node will check whether it is the first time for receiving the message. If yes, the node will receive and forward the message timely. If not, receives it without forwarding. If $\operatorname{dis}(\operatorname{Node}(i), \mathrm{CH}) \leq \operatorname{dis}\left(\right.$ Forward $\left._{\text {latest }}, \mathrm{CH}\right)$, the node will drop the packet. By using the direction based broadcasting algorithm, messages could be transmitted along two certain directions, which are upstream and downstream directions of the road. Meanwhile, each node only has one chance to forward a message. Data redundancy can be reduced.

After the establishment of the cluster, cluster head broadcasts its Declaration-Message, cluster members receive it and forward it. When a cluster member receives the message, it will record the information of the cluster head and forwarding nodes. If further forwarding is permissible, the member node adds its ID and position into the message and meanwhile sets the value of $\mathrm{K}$ plus 1 . Forwarding is terminated in case the message is beyond the geographic scope of the current road section. At the beginning, we have made a supposition that links are bidirectional, so when the message broadcasting finishes, all members will establish routings to the cluster head according to forwarding nodes information.

\section{B. CM Status Data Collection, Fusion and Broadcasting}

Cluster members send their expected status data to the cluster head by the maintained routing before $T_{0}$ timestamp, which consists in the Declaration- Message. The status data is included in the Status- Message, of which the format is designed as follows:

$$
<I D_{-} C M, v_{0}, L_{0}, \text { Time }_{0}>
$$


Where $I D_{-} C M$ is the member's ID, $v_{0}$ is the expected speed at $T_{0}, L_{0}$ is the expected location at $T_{0}$, Time denotes the expected travel time. The three status index are calculated by formula (1).

$$
\left\{\begin{array}{l}
v_{k}=v_{c}+a\left(T_{k}-T_{c}\right) \\
L_{k}=L_{c}+v_{c}\left(T_{k}-T_{c}\right)+\frac{1}{2} a\left(T_{k}-T_{c}\right)^{2} \\
\text { Time }_{k}=\frac{L_{k}-L_{\text {end }}}{v_{k}}
\end{array}\right.
$$

Where $T_{c}$ is the time when a member sends its status data. $v_{c}$ is the current speed at $T_{c}$, and $L_{c}$ is the current location at $T_{c} . L_{\text {end }}$ denotes the endpoint location of the road section. Let the value of $k$ is 0 , and expected status information at $T_{0}$ will be obtained.

The cluster head receives messages sent by the members and builds a list of its members. Then it calculates the average speed of the cluster and the next timestamp $T_{1}$, which is calculated by formula (2).

$$
T_{k+1}=T_{k}+\sigma_{k}, \quad \sigma_{k}=\frac{\mu}{\bar{v}}
$$

Where ${ }^{\mu}$ is constant. $\sigma_{k}$ denotes the time interval between the current timestamp and the next timestamp. From formula (2), we can realize that the higher the average speed is, the shorter time interval two adjacent timestamps alternate, and the more frequently the cluster head broadcasts management messages to members. This can better adapt the topological changes in a high speed moving scene. On the contrary, the lower the average speed is, the less frequently cluster head broadcasts. When the topology changes slowly, it will reduce cluster maintaining cost. Dynamic timestamps based on average speed of the cluster can better adapt the high variable speed environment. Since speed of vehicles on road is limited, the value of $\sigma_{k}$ can be considered within a certain range, and make $\sigma_{k} \in\left[\sigma_{\min }, \sigma_{\max }\right]$. The maximum delay time mentioned in the initial cluster head selection stage in Section 3 is exactly the $\sigma_{\max }$ given here.

Let the value of $k$ be 0 , and then $T_{1}$ timestamp will be obtained.

At $T_{0}$ timestamp, by using the direction-based broadcasting algorithm, the cluster head broadcasts the Management-Message which contains the status information of the cluster and the next timestamp $T_{1}$. The format of the Management-Message is designed as follows:

$$
<I D_{-} \mathrm{CH}, L_{-} \mathrm{CH}, \mathrm{ID} \mathrm{F}_{-} \mathrm{FW}, L_{-} \mathrm{FW}, \mathrm{T}_{0}, \mathrm{~K}, \mathrm{CS}>
$$

Comparing to the Declaration-Message, it adds a table of cluster status $C S$, which contains the following information:

$$
C S:<\bar{v}, v_{\min }, v_{\max }, \text { Time }_{\min }, \text { Time }_{\max }>
$$

Where $\bar{v}$ is the average speed, $v_{\min }$ is the minimum speed, $v_{\max }$ is the maximum speed, Time ${ }_{\min }$ is the minimum travel time, and Time $_{\max }$ is the maximum travel time.
Each cluster member updates its routing table according to the latest management message broadcasted by the cluster head and carries out the next round of data transmission.

\section{Cluster Maintenance Strategy Based on Dynamic Timestamps}

The cluster head invariably broadcasts the management message containing the next timestamp information at the current timestamp. Because the status of each member is prediction based, meanwhile the broadcast time is constantly changing associated with vehicle speed, maintenance demands caused by nodes movement could be satisfied in a cluster. In this part, we introduce other two maintenance cases, which are cluster head disappearing and new nodes joining.

\section{Cluster head disappears}

Under each of below two conditions a cluster head may disappear: the cluster head vehicle moves out from the scope of the current cluster, or links between the cluster head and members are interrupted. No matter what the situation is, as a cluster member, it cannot receive the cluster head's management message on time. Suppose the cluster head moves out from the scope or there happens to be link interruption in the time range $T_{k} \sim T_{k+1}$, that is to say, cluster members can receive management message at $T_{k}$, but can't at $T_{k+1}$. The reconfiguration of cluster head is described as follows.

Cluster member which cannot receive management message on time calculates a weight value. In order that the selected cluster head can serve for a longer time and have higher relative stability among members, two indexes are chosen to describe the weight value: travel time and speed difference. The weight value is calculated by formula (3).

$$
\begin{aligned}
\omega(i)= & \alpha \cdot \omega_{\text {Time }}+\beta \bullet \omega_{\text {Speed }} \\
= & \alpha \cdot \frac{\text { Time }_{k}(i)-\text { Time }_{\min }}{\text { Time }_{\max }-\text { Time }_{\min }}+ \\
& \beta \cdot\left(1-\frac{\left|v_{k}(i)-\bar{v}\right|}{\max \left(\left|v_{\max }-\bar{v}\right|,\left|v_{\min }-\bar{v}\right|\right)}\right)
\end{aligned}
$$

Where $\omega_{\text {Time }}$ and $\omega_{\text {Speed }}$ denote the service time and the stability. $\alpha, \beta$ are weight coefficients, $\alpha+\beta=1$. Formula (3) gives the final dimensionless form of the two factors, which are unified according to the cluster status information contained in the latest management message. The weight value calculated by formula (3) is in the range of $0-1$.

Members delay a period of time $T_{\text {Delay }}(i)$. If the member doesn't receive any declaration message or management message from other cluster head during this period of time, it will broadcast its declaration message and compete for cluster head. The delay time $T_{\text {Delay }}(i)$ is related to the weight value and calculated by formula (4).

$$
T_{\text {Delay }}(i)=(1-\omega(i)) \cdot \sigma_{\max }
$$

A node which has longer travel time and is closer to the average speed obtains a higher weight value. The node which has the maximum weight value broadcasts declara- 


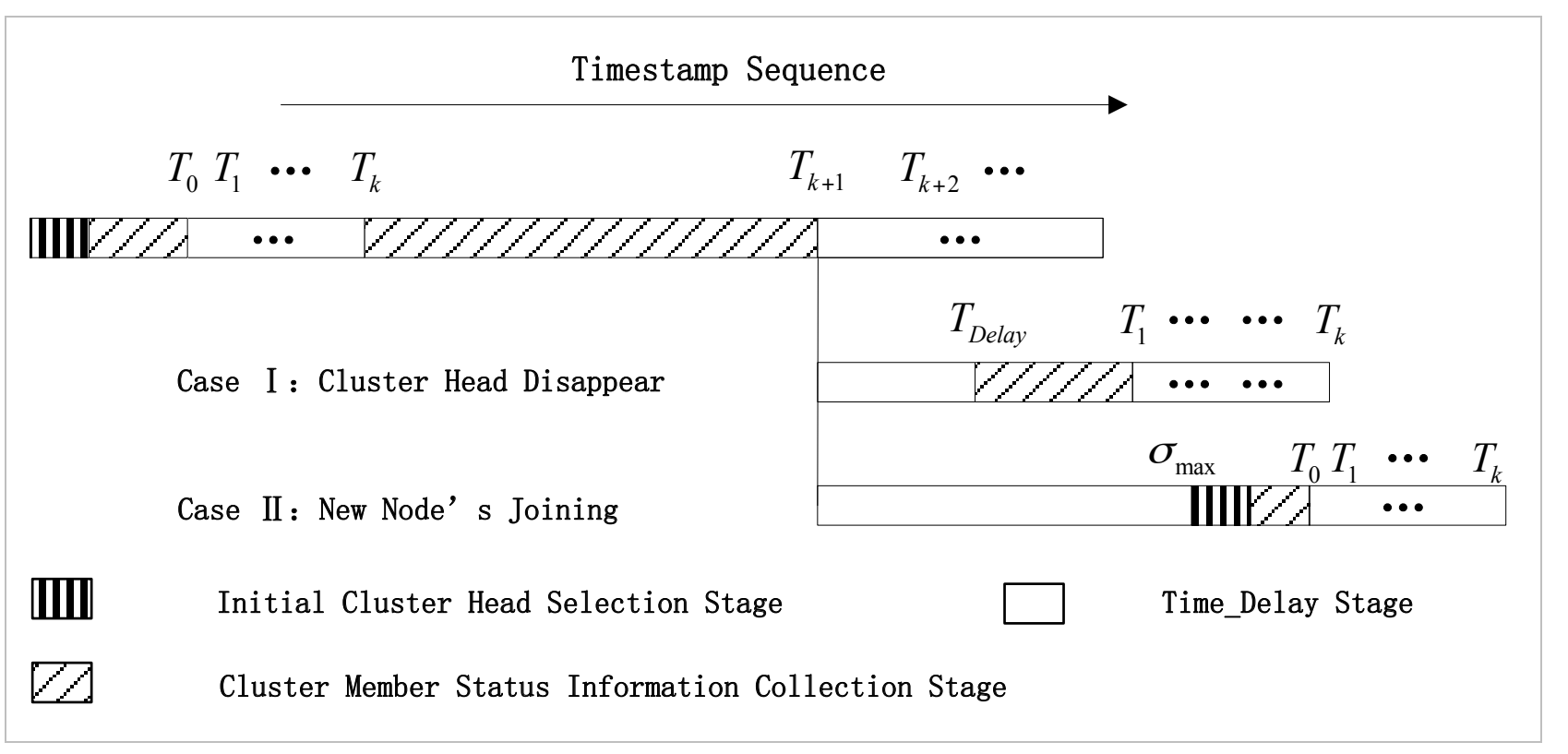

Figure 2. Selection and maintaining of cluster head based on timestamp sequences

tion message at the earliest so that it will be selected as a cluster head.

\section{New nodes join}

When a node joins in the cluster, it clears the status data of the former cluster and waits for a time interval $\sigma_{\max }$. During the period of time interval, if the new node receives management message of the new cluster from cluster head, it stores the relevant data, sends excepted status message and waits for the next message. If it doesn't receive the message, competes for the cluster head following the 'First Declare Win' principle.

The selection and maintaining of cluster head based on dynamic timestamps can be shown as Figure 2 .

Figure. 2 shows the selection and maintaining process of cluster head based on dynamic timestamps. At the beginning, initial cluster head was selected following the 'Randomly Competition, First Declare Win' principle. The cluster head broadcasts management message at each timestamp. During the cluster member status information collection stage, cluster members send their excepted status messages to the cluster head, and the messages will be processed by the cluster head. When the cluster head disappears or new node joins, different strategies will be taken to update cluster head so that the cluster structure can be well maintained.

\section{Clustering Principles And Performance ANALYSIS}

\section{A. Some Other Clustering Principles}

In order to improve reliability of the algorithm, in this section, we propose some clustering principles, including multiple cluster heads principle, cluster heads screening principle and cluster scale partition principle.

\section{Multiple cluster heads principle}

Due to the movement of vehicles on roads, nodes in the cluster may be distributed in several communication isolated sub-clusters, such as intermittent traffic flow. There is no forwarding node between two adjacent sub-clusters. The management messages will not be transmitted to all nodes in the cluster since communication holes appear. When this situation happens, multiple cluster heads are permitted. Cluster head is selected in each sub-cluster, and each head is responsible for the management of its subcluster.

\section{Cluster heads screening principle}

Due to the movement of vehicles on roads, nodes in communication isolated sub-clusters may merge into a cluster. Then nodes in the cluster will receive different management messages from different cluster heads in the same cluster. If there are at least two different messages broadcasted, nodes calculate the weight values of the cluster heads according to formula (3), the biggest value node will keep the state of cluster head and the rest change their roles to cluster members.

\section{Cluster scale partition principle}

In our algorithm, the cluster is established on a certain road section. The scale of the cluster is exactly the scale of the divided road section. It is affected by the data processing ability of a vehicle and the characteristics of traffic flow on the road. The scale of a cluster should not be too large. On one hand, status data fusion will not be finished timely when there are too many nodes in the cluster, for the reason that the processing ability of the cluster head is limited. On the other hand, hop count of link will increase if the road section is too long, resulting in obvious influence on the algorithm caused by forwarding delay. Meanwhile, the scale should not be too small. Otherwise, it will bring too many clusters in the network and much too frequent updates of cluster heads.

\section{B. Performance Analysis}

In the PSCA, clusters are established on certain road sections according to position information. Clustering process is simple and easy to realize. The PCSA can be classified to multi-hop clustering algorithm, which can effectively decrease the number of clusters in the network.

Cluster members don't need to maintain the information of their neighbors so they don't have to broadcast hello messages periodically. The maintenance of a cluster is realized through management messages broadcasted by 
the cluster head. Dynamic timestamps are also introduced into the maintenance strategy. It can greatly reduce the establishment and maintenance cost. When there is need for data transmission in some applications, for example, traffic information collection in CVIS, cluster members' status data can be attached in the application messages, so that maintenance cost can be further reduced.

The proposed PSCA follows multiple cluster heads principle and cluster heads screening principle. It can better adapt the high variable topology structure caused by environment of vehicles.

\section{Simulations And Results}

In this section, we study the performance of PSCA. In order to make a comparison with the traditional single hop VANET clustering algorithms, the classical Lowest-ID algorithm is selected to be compared on two indexes, cluster heads number and data packages number. The road conditions and the vehicle kinematical model are complied by $\mathrm{VC}++$. All simulations are performed using network simulator NS-2. The cluster is established on a $1000 \mathrm{~m}$ long road section with two lanes. Vehicles enter into the section from the starting point, and time intervals between two adjacent vehicles follow Poisson distribution with parameter $\lambda$. The speed of a vehicle changes from $(1-20 \%) \cdot \bar{v}$ to $(1+20 \%) \cdot \bar{v}$, where $\bar{v}$ is the average speed and changes from $20 \mathrm{~km} / \mathrm{h}$ to $60 \mathrm{~km} / \mathrm{h}$. The density of vehicles distributing on the road can be changed through the adjustment of parameters $\lambda$ and $\bar{v}$. Suppose the clustering algorithms are available if $2 \mathrm{~s}$ is taken as the clustering broadcast period at the highest speed $(60 \mathrm{~km} / \mathrm{h})$. Other parameters that our simulation need are shown in Tab.1.

TABLE I.

SIMULATION PARAMETERS

\begin{tabular}{|l|l|}
\hline \multicolumn{1}{|c|}{ Parameter } & \multicolumn{1}{c|}{ Value } \\
\hline Length of road section & $1000 \mathrm{~m}$ \\
\hline Number of lanes & 2 \\
\hline MAC protocol & IEEE $802.11 \mathrm{p}$ \\
\hline MAC data transfer rate & $20 \mathrm{Mb} / \mathrm{s}$ \\
\hline Size of data package & $2 \mathrm{~Kb}$ \\
\hline Communication range & $100 \sim 500 \mathrm{~m}$ \\
\hline Vehicle density & $20 、 50 、 100 \mathrm{veh} / \mathrm{km}$ \\
\hline Average speed of vehicles & $20 \sim 60 \mathrm{~km} / \mathrm{h}$ \\
\hline Speed variation for a single vehicle & $\pm 20 \%$ \\
\hline Minimum broadcast period & $2 \mathrm{~s}$ \\
\hline
\end{tabular}

\section{A. Number of Cluster Heads Simulation}

Make an adjustment of the vehicles density on the road and set the values as $100 \mathrm{veh} / \mathrm{km}$ 、50veh $/ \mathrm{km} 、 20 \mathrm{veh} / \mathrm{km}$. The broadcasting period for PSCA and Lowest-ID are both $2 \mathrm{~s}$. Each simulation lasts for $10 \mathrm{~min}$. Figure out the number of cluster heads under different densities of vehicles and different communication ranges, as is shown in Figure.3.

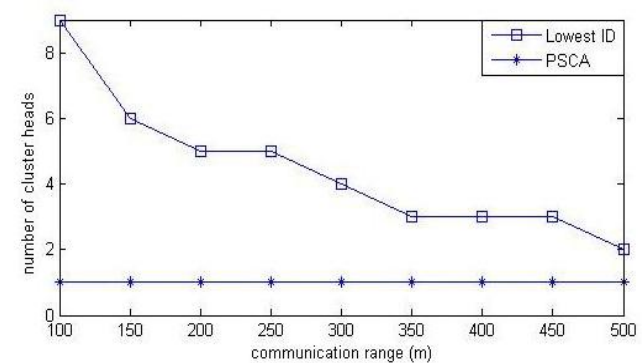

(a)Vehicle density $=100 \mathrm{veh} / \mathrm{km}$

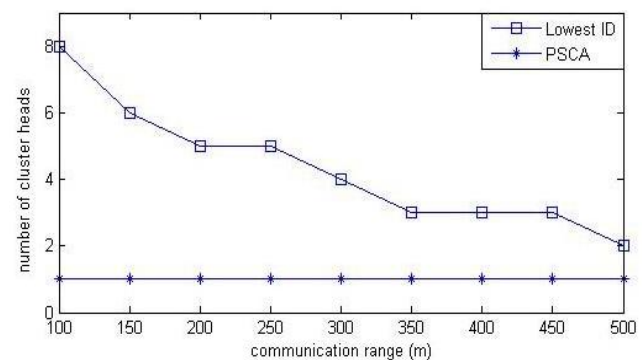

(b) Vehicle density $=50 \mathrm{veh} / \mathrm{km}$

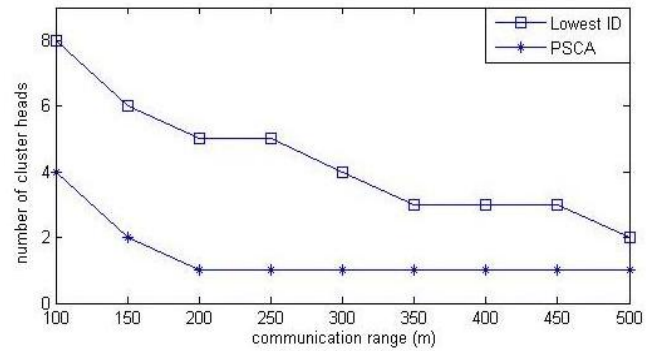

(c) Vehicle Density $=20 \mathrm{veh} / \mathrm{km}$

Figure 3. Number of cluster heads under different communication ranges and vehicle densities

As the communication range increases, the number of cluster heads decreases. PSCA keeps only one cluster head when the vehicle density is high, while the LowestID algorithm generates a large number of cluster heads. When the vehicles are spare, PSCA also generates multiple cluster heads, but far too less than the Lowest-ID algorithm at the same communication range. The reason is that PSCA selects cluster heads according to the connectivity. Only when the nodes are spare and communication isolated sub-clusters appear, new cluster head can be generated. For this reason, the number of cluster heads in the network is effectively limited.

\section{B. Number of Data Packages Simulation}

Make an adjustment of the vehicles density on road and set the values as $100 \mathrm{veh} / \mathrm{km} 、 50 \mathrm{veh} / \mathrm{km} 、 20 \mathrm{veh} / \mathrm{km}$. Simulations are carried out under different average speed of vehicles. Each simulation lasts for 30 s. In order to ensure the comparability with the Lowest-ID algorithm, $2 \mathrm{~s}$ is taken as the clustering broadcast period for both algorithms at the highest speed.

Figure out the average number of the data packages after 20 independent experiments under different densities of vehicle and different average speed. The results are shown in Figure.4. 


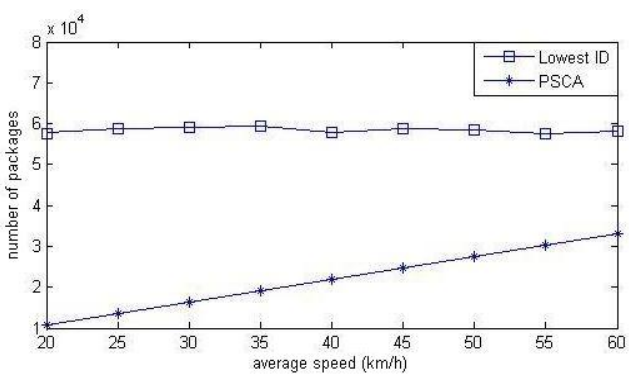

(a)Vehicle density $=100 \mathrm{veh} / \mathrm{km}$

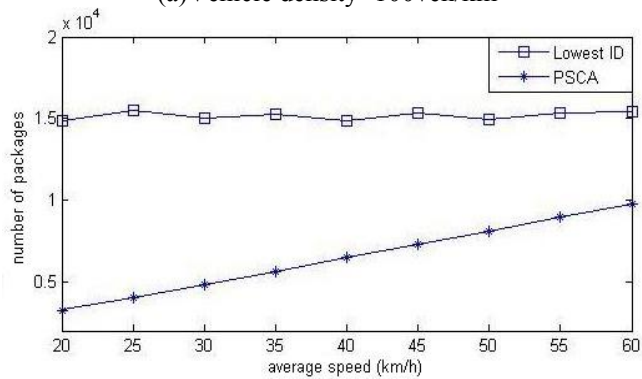

(b) Vehicle density $=50 \mathrm{veh} / \mathrm{km}$

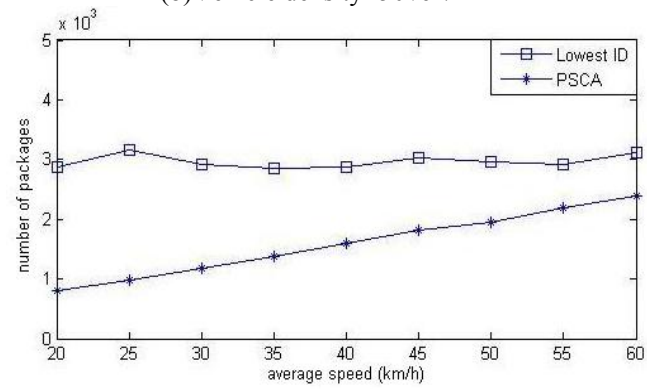

(c) Vehicle density $=20 \mathrm{veh} / \mathrm{km}$

Figure 4. Number of data packages under different speed and vehicle densities

In Fig.4, numbers of clustering data packages are presented in different situations. It is clear that the number of data packages created by PSCA is much lower than the Lowest-ID algorithm. There are two reasons. On one hand, in PSCA, the cluster sends cluster management messages using the direction based broadcast algorithm, while the members transmit their status messages by multi-hop unicast routing. There is no need for each node broadcasting hello message to its neighbors and maintaining a table of neighbors. On the other hand, traditional single hop algorithms broadcast clustering messages at a defined period, so the establishment and maintenance cost of clustering over the same time interval is almost constant. PSCA carries out a dynamic timestamps-based maintenance strategy, in which broadcasting period can be adjusted according to the characteristic of traffic flow. When the speed is low, broadcasting period will be extended. It benefits the reduction of clustering data packages.

\section{CONCLUSIONS AND FUTURE WORK}

Based on the full use of GPS and its time synchronism service, the position sensitive clustering algorithm for VANET has been proposed. Compared to the traditional single hop clustering algorithms, it performs well on the index of cluster heads number and clustering overhead. It is significant for the hierarchical routing research and some other CIVS applications in VANET.
The PSCA is proposed on the basis that the scale of the cluster is reasonable. Although we have given the cluster scale partition principle, concrete cluster size model is still lacked. The cluster partition according to the data processing capability of vehicles and the influences on the algorithm caused by forwarding delay will be further studied.

\section{REFERENCES}

[1] A. Nandan, S. Das, G. Pau, M. Gerla, and M. Y. Sanadidi, "Cooperative downloading in vehicular ad-hoc wireless networks," in Proceedings Second Annual IEEE Conference on Wireless Ondemand Network Systems and Services (WONS'05), St. Moritz, Switzerland, pp. 32-41, 2005. http://dx.doi.org/10.1109/WONS. 2005.7

[2] P. Zhou, T. Nadeem, P. Kang, C. Borcea, and L. Iftode, "EZCab: A cab booking application using short-range wireless communication," in Proceedings 3rd IEEE International Conference on Pervasive Computing and Communications, pp. 27-38, 2005. http://dx.doi.org/10.1109/PERCOM.2005.21

[3] D Wu, J Cao, Y Ling, J Liu, and L Sun, "Routing algorithm based on multi-community evolutionary game for VANET," Journal of Networks, vol.7, no.7, pp. 1106-1115, July 2012. http://dx.doi.org/10.4304/jnw.7.7.1106-1115

[4] [4] M. S. Almalag, and M. C. Weigle, "Using traffic flow for cluster formation in vehicular ad-hoc networks," The 35th Annual IEEE Conference on Local Computer Networks, pp. 10-14, October 2010 .

[5] M. D. Venkata, M. M. M. Pai, R. M. Pai, and J. Mouzna, "Traffic monitoring and routing in VANETs-a clustering based approach," 2011 11th International Conference on ITS Telecommunications, pp. 24-32, 2011.

[6] J Li, and S Jiang, "A scalable clustering algorithm in dense mobile sensor networks," Journal of Networks, vol.6, no.3, pp. 505-512, March 2011. http://dx.doi.org/10.4304/jnw.6.3.505-512

[7] T. C. Hou, and T. J. Tsai, "An access-based clustering protocol for multihop wireless ad hoc networks," IEEE Journal on Selected Areas in Communications, vol.19, no.7, pp. 1201-1210, 2001. http://dx.doi.org/10.1109/49.932689

[8] D. Kumar, T. Aseri, and R. B. Patel, "EEHC: Energy Efficient Heterogeneous Clustered Scheme for Wireless Sensor Networks", Computer Communications, vol.32, no.4, pp. 662-667, March 2009. http://dx.doi.org/10.1016/j.comcom.2008.11.025

[9] M. Gerla, and J. T. C. Tsai, "Multicluster, mobile, multimedia radio network," Wireless Networks,vol.1, no.3, pp. 255-65, 1995. http://dx.doi.org/10.1007/BF01200845

[10] F. Peng, J. Haran, J. Dillenburg, and P. C. Nelson, "Traffic model for clustering algorithms in vehicular ad-hoc networks," Proceedings of the Consumer Communications and Networking Conference, pp. 168-172, 2006.

[11] M. Chatterjee, S. K. Das, and D. Turgut, "WCA: A weighted clustering algorithm for mobile ad hoc networks," Computing IEEE, vol. 5, no.2, pp. 193 204, 2002.

[12] R. T. Goonewardene, F. H. Ali, and E. Stipidis, "Robust mobility adaptive clustering scheme with support for geographic routing for vehicular ad hoc networks," Intelligent Transport Systems, IET,vol.3, no.2, pp. 148-158, 2009. http://dx.doi.org/10.1049/ietits:20070052

[13] N. Maslekar, M. Boussedjra, J. Mouzna and L. Houda, "Direction based clustering algorithm for data dissemination in vehicular networks," Vehicular Networking Conference (VNC), 2009 IEEE, pp. 1-6, February 2010.

[14] Z. G. Wang, L. C. Liu, M. C. Zhou, and N. Ansari, “A positionbased clustering technique for ad hoc intervehicle communication," IEEE Transaction on System, Man, and Cybernetics, Part C: Applications and Reviews, vol.38, no.2, pp.201-208, 2008. http://dx.doi.org/10.1109/TSMCC.2007.913917

[15] I. Salhi, S. M. Senouci, and M. Cherif, "A new framework for data collection in vehicular networks," IEEE ICC'2009 Dresden, pp. 14-18, 2009. 


\section{SPECIAL FOCUS PAPER \\ A Position SEnsitive Clustering Algorithm For VANET}

[16] S. Yang, J. Wu, and J. N. Cao, "Connected k-hop clustering in ad hoc networks," International Conference on Parallel Processing, ICPP 2005, pp. 373-380, 2005

[17] G. Angione, P. Bellavista, A. Corradi, and E. Magistretti, "A khop clustering protocol for dense mobile ad-hoc networks," 26th IEEE International Conference on Distributed Computing Systems Workshops, pp. 10-16, 2006.

[18] J. Y. Yu, and P. H. J. Chong, "An efficient clustering scheme for large and dense mobile ad hoc networks (MANETs)", Computer Communications, vol.30, pp. 5-16, Aug 2006. http://dx.doi.org/10.1016/j.comcom.2006.07.014

\section{AUTHORS}

H.Q. Liu is currently enrolled for joint courses for master and doctor degrees in School of Control Science and Engineering, Shandong University, Jinan, 250061, Shandong, China. His research interests include intelligent transportation system, vehicular ad hoc network and cooperative vehicle infrastructure system (e-mail: hqliu623@gmail.com).

L.C. Yang* is currently a professor at School of Control Science and Engineering in Shandong University, Jinan, 250061, Shandong, China. His research interests include artificial intelligence and intelligent control, control theory and applications, intelligent transportation systems, and vehicular ad hoc networks. In this paper, he is the corresponding author (* corresponding author, e-mail: yanglc@sdu.edu.cn).

Y. Zhang is currently a Ph.D candidate in School of Control Science and Engineering, Shandong University, Jinan, 250061, Shandong, China. He is also an associate professor at School of Mechanical, Electrical and Information Engineering, Shandong University at Weihai. His research interests include intelligent transportation system, computer network and wireless communication (e-mail: zhangyao@sdu.edu.cn).

$\mathbf{L}$. Wu is currently enrolled for joint courses for master and doctor degrees in School of Control Science and Engineering, Shandong University, Jinan, 250061, Shandong, China. His research interests include intelligent transportation system and cooperative vehicle infrastructure system (e-mail: wulei-17@163.com).

Submitted 28 August 2013. Published as re-submitted by the authors 23 January 2014. 